\title{
Prolonged QTc Interval and
} Polymorphic Ventricular Tachycardia in a Patient with Multiple Myelomaassociated Cardiac Amyloidosis

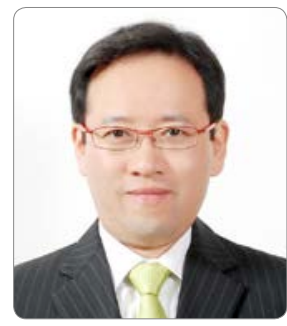

Jin-Seok Kim, MD, PhD

Divison of Cardiology, Department of Internal Medicine, Korea University Ansan Hospital, Ansan, Korea

Received: June 22, 2015

Revision Received: June 22, 2015

Accepted: December 15, 2015

Correspondence: Jin-Seok Kim, MD, PhD

Clinical Associate Professor

Division of Cardiology

Department of Internal Medicine Korea University Ansan Hospital 123, Jeokgum-ro, Danwon-gu, Ansan-si, Gyeonggi-do, Republic of Korea,

425-707

Tel. +82-31-8099-6349, Fax. +82-31-8099-6352

E-mail: heartmania@unitel.co.kr or heartmania@ nate.com

Copyright (C) 2015 The Official Journal of Korean Heart Rhythm Society Editorial Board \& MMK Co., Ltd.

\begin{abstract}
Cardiac amyloidosis is associated with systemic amyloidosis and patients usually presents with symptoms of congestive heart failure and echocardiographic evidence of restrictive cardiomyopathy. The disease progresses rapidly, and the prognosis is usually poor because patients often present with nonspecific signs and symptoms, which can delay an accurate diagnosis. Although cardiac arrest due to ventricular arrhythmias is responsible in some cases, ventricular arrhythmias in cardiac amyloidosis are uncommon. The present report describes an unusual case of a 52-year-old woman who had been diagnosed as having multiple myeloma and presented with a long QT interval and polymorphic ventricular tachycardia. These findings may be associated with disease activity.
\end{abstract}

Key Words: - Amyloidosis -Multiple myeloma -Tachycardia Ventricular • Long QT Syndrome

\section{Introduction}

Systemic amyloid light chain (AL) amyloidosis (previously known as primary amyloidosis) is a multisystem disease characterized by the extracellular deposition of fibrils composed of immunoglobulin light chains within various organs. $\mathrm{AL}$ amyloidosis is associated with different types of monoclonal plasma cell dyscrasias, including multiple myeloma and other monoclonal gammopathies. ${ }^{1}$ Cardiac involvement is clinically reported in approximately one-half of all cases, but the heart is affected pathologically in up to $90 \% \mathrm{AL}$ amyloidosis patients. ${ }^{2,3}$ Patients with cardiac amyloidosis can present a wide spectrum of clinical manifestations, but usually have symptoms of congestive heart failure and echocardiographic evidence of restrictive cardiomyopathy. The present report describes an unusual case of multiple myeloma-associated cardiac amyloidosis in a patient who presented a long QT interval (QTc) and polymorphic ventricular tachycardia (VT).

\section{Case}

A 52-year-old woman was admitted to the hospital owing to exertional dyspnea with atypical chest discomfort. She had been well previously and had no prior cardiovascular disease or 
complaints. Physical examination revealed mild bilateral lower extremity edema and bilateral small pleural effusions on chest radiography. Initial electrocardiogram (ECG) showed left anterior fascicular block; a QS complex in leads III, aVF, and $\mathrm{V}_{1}$ to $\mathrm{V}_{3}$; and a normal QTc interval of $437 \mathrm{msec}$ (Figure 1). A transthoracic echocardiogram showed severe left ventricular (LV) hypertrophy with an interventricular septal wall thickness of 19.5 $\mathrm{mm}$, a small posterior pericardial effusion, LV ejection fraction, and a restrictive filling pattern (Figure 2A). Cardiac magnetic resonance with gadolinium contrast showed asymmetric LV thickening with diffuse subendocardial enhancement on delayed images, suggesting infiltrative cardiomyopathy (Figure 2B). Urine and serum studies (Figure 3) revealed an isolated band of electrophoretic mobility in the $\lambda$ lane (serum $\lambda$ immunoglobulin level, $511.5 \mathrm{mg} / \mathrm{L}$; normal range, $5.51-26.3 \mathrm{mg} / \mathrm{L}$ ). Results of immunofluorescence analysis were strongly positive for $\lambda$ and weakly positive for $\kappa$ protein, indicative of $\lambda(\mathrm{AL})$ amyloidosis. $\mathrm{A}$ bone marrow biopsy showed increased plasma cells with $\lambda$ light chain, and flow cytometry yielded $\lambda$-restricted, CD138, and MUM-1, consistent with a diagnosis of multiple myeloma.

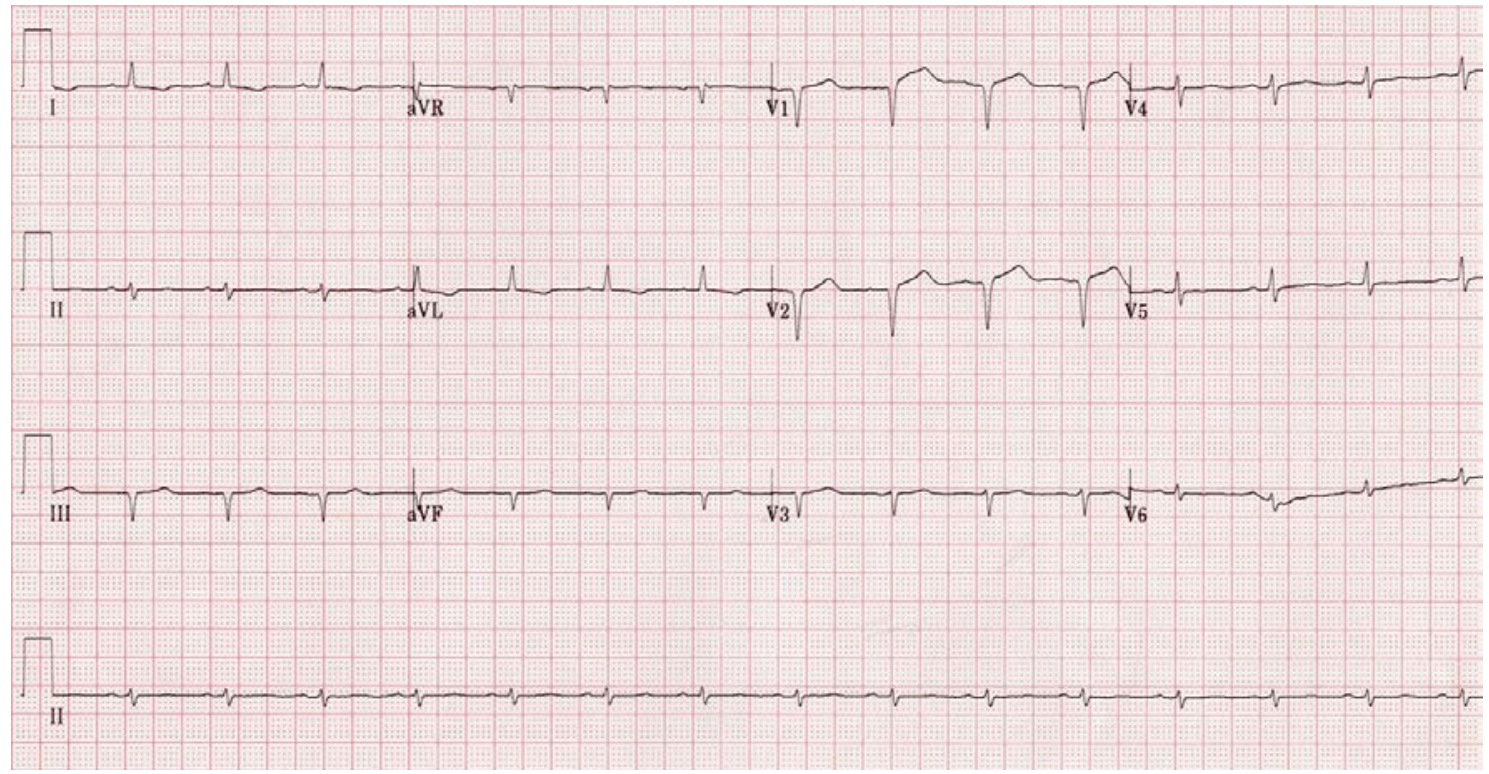

Figure 1. Initial electrocardiogram shows a low voltage in both limb and precordial leads, left anterior fascicular block, pseudo-infarct QS waves in leads III, aVF, V1, and V2, and a normal QTc interval.

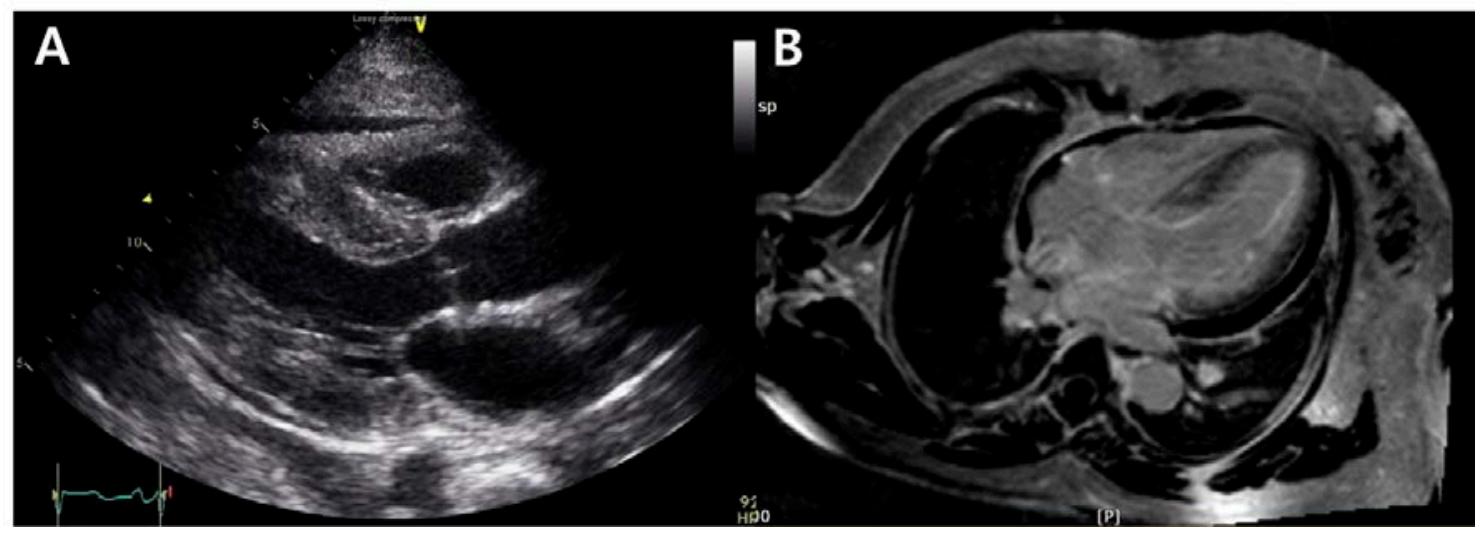

Figure 2. (A) Echocardiogram taken from the parasternal long-axis view shows severely thickened left ventricular walls with mild pericardial effusion. (B) Cardiac magnetic resonance imaging represents delayed gadolinium uptake with a diffuse and predominantly subendocadial distribution pattern, suggesting cardiac amyloidosis. 
A

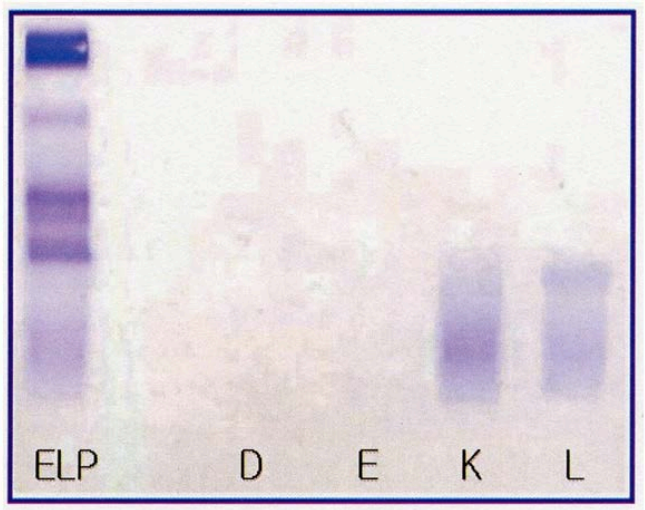

B

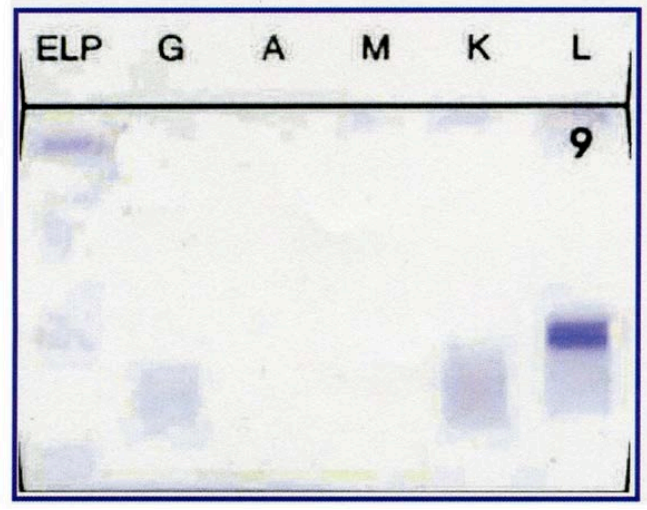

Figure 3. Immunofixation electrophoresis for serum (A) and urine (B) shows monoclonal gammopathy ( $\lambda$ type).

Endocardial biopsy could not be performed for confirming the diagnosis of cardiac amyloidosis because of the patient's refusal. The patient was started on low-dose furosemide and oncologic treatment with thalidomide and dexamethasone for AL amyloidosis and plasma cell myeloma.

Three weeks later, after completing the first cycle of chemotherapy, she was readmitted for recurrent episodes of syncope. Even though biochemical evaluations revealed normal renal function (creatinine level, $1.24 \mathrm{mg} / \mathrm{dL}$ ) with normal levels of serum electrolytes, her ECG showed significantly low voltages and a markedly prolonged QTc of $537 \mathrm{msec}$ (Figure 4A), longer than that on the previous ECG. Recurrent episodes of self-limiting polymorphic VT were documented on 24-h Holter monitoring at the time of syncopal attack (Figure 4B). To shorten QTc interval, transvenous overdrive pacing was performed and its pacing rates were maintained at 90 to 110 beats/min. After short-term use of temporary pacing, isoproterenol was administered intravenously and its dosage was titrated to maintained basal heart rate above 60 beats $/ \mathrm{min}$. With the second cycle of chemotherapy for myeloma, she had no recurrent polymorphic VT, even though the QTc remained at $504 \mathrm{msec}$.

\section{Discussion}

Amyloidosis usually involves more than one organ system, in which anti-parallel $\beta$-pleated sheets of amyloid fibrils are deposited. AL amyloidosis is the most common form that causes cardiac amyloidosis in patients with plasma cell myeloma, as in this report. ${ }^{4}$ Cardiac amyloidosis is common in patients with $\mathrm{AL}$ amyloidosis. Infiltration of the heart by amyloid proteins results in biventricular wall thickening, resulting in restrictive cardiomyopathy that manifests at a later stage with heart failure and conduction abnormalities. $^{5}$

Cardiac amyloidosis should be suspected in any patient who presents with heart-failure symptoms, low ECG voltage, and a restrictive filling pattern or ventricular hypertrophy on echocardiography. ${ }^{2}$ The disease progresses rapidly, and the prognosis is usually poor because patients often present with nonspecific signs and symptoms that prolong the time to diagnosis. Up to $30 \%$ patients die suddenly because of pulseless electrical activity or electromechanical dissociation. ${ }^{67}$ Although cardiac arrest due to ventricular arrhythmias is responsible in some cases, ${ }^{8}$ ventricular arrhythmias in cardiac amyloidosis are uncommon.

The present case of cardiac amyloidosis had a prolonged QTc and polymorphic VT. The progression in QTc interval with subsequent ventricular arrhythmias might have correlated with the disease's progression. Cardiac amyloidosis should be considered in patients who present with ventricular arrhythmias, especially when low voltages are observed on ECG and when infiltrative heart disease is strongly suspected.

\section{References}

1) Halwani O, Delgado DH. Cardiac amyloidosis: An approach to diagnosis and management. Expert Rev Cardiovasc Ther. 2010;8:1007-1013. 
$\mathbf{A}$

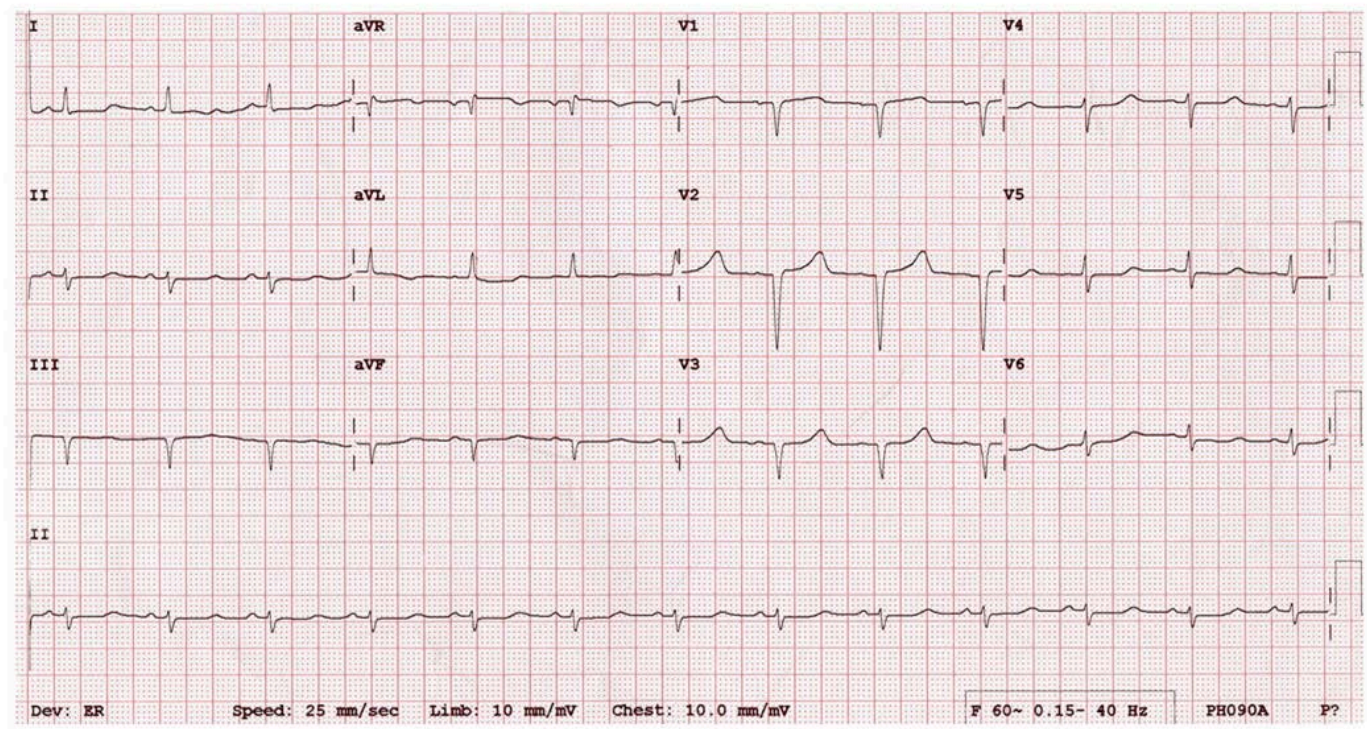

B

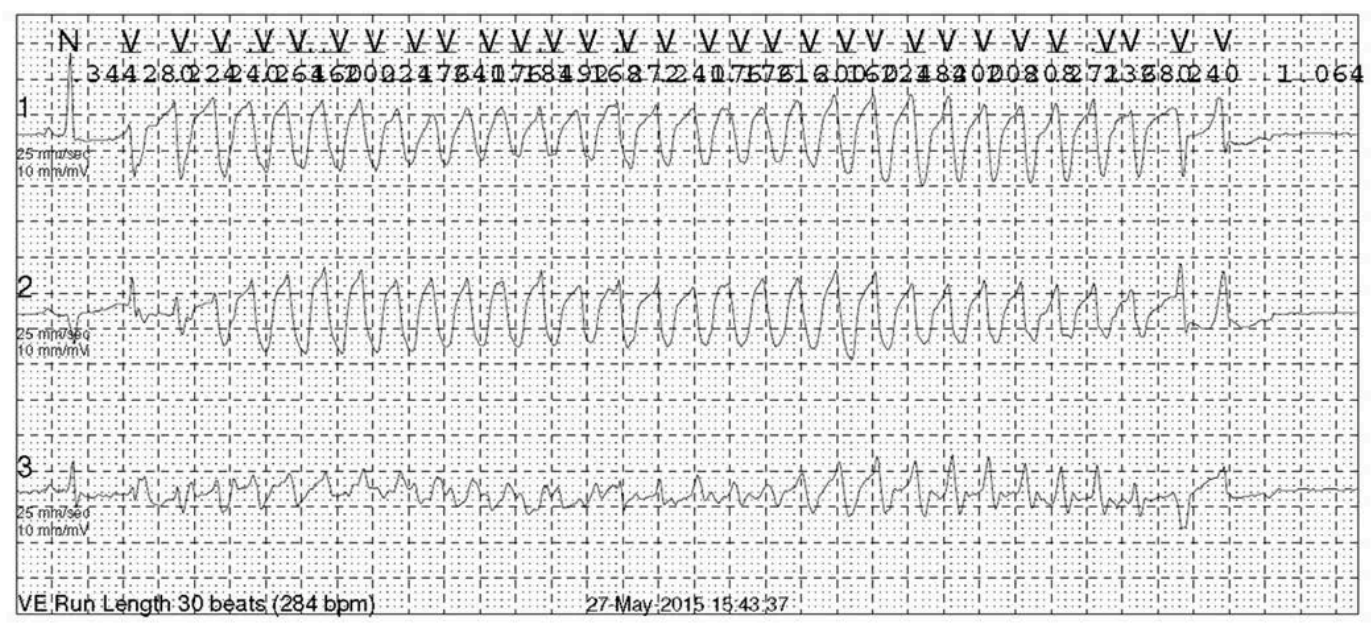

Figure 4. (A) Electrocardiogram on readmission shows low voltage in the limb leads and a prolonged QTc interval of 537 msec. (B) 24-hour Holter recording reveals polymorphic ventricular tachycardia induced by ventricular ectopy.

2) Falk RH, Dubrey SW. Amyloid heart disease. Prog Cardiovasc Dis. 2010;52:347-361.

3) Selvanayagam JB, Hawkins PN, Paul B, Myerson SG, Neubauer S. Evaluation and management of the cardiac amyloidosis. J Am Coll Cardiol. 2007;50:2101-2110.

4) Dubrey SW, Hawkins PN, Falk RH. Amyloid diseases of the heart: Assessment, diagnosis, and referral. Heart. 2011;97:75-84.

5) Shah KB, Inoue Y, Mehra MR. Amyloidosis and the heart: A comprehensive review. Arch Intern Med. 2006;166:1805-1813.

6) Seethala S, Jain S, Ohori NP, Monaco S, Lacomis J, Crock F, Nemec J. Focal monomorphic ventricular tachycardia as the first manifestation of amyloid cardiomyopathy. Indian Pacing Electrophysiol J.2010;10:143-147.

7) Dhoble A, Khasnis A, Olomu A, Thakur R. Cardiac amyloidosis treated with an implantable cardioverter defibrillator and subcutaneous array lead system: Report of a case and literature review. Clin Cardiol. 2009;32:E63-65.

8) Kristen AV, Dengler TJ, Hegenbart U, Schonland SO, Goldschmidt H, Sack FU, Voss F, Becker R, Katus HA, Bauer A. Prophylactic implantation of cardioverter-defibrillator in patients with severe cardiac amyloidosis and high risk for sudden cardiac death. Heart Rhythm. 2008;5:235-240. 